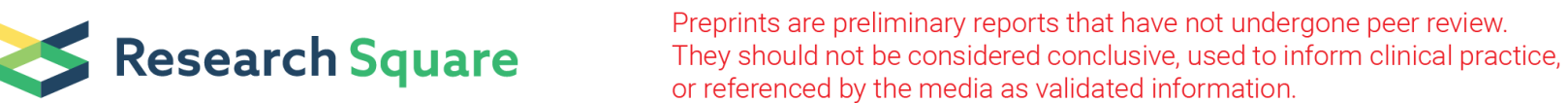

\section{A five-step process for designing implementable complex interventions: A worked example using the Tailored intervention for ANxiety and DEpression Management (TANDEM) for patients with chronic obstructive pulmonary disease (COPD)}

Liz Steed ( $\nabla$ e.a.steed@qmul.ac.uk)

Queen Mary University of London https://orcid.org/0000-0003-1926-3196

Karen Heslop-Marshall

Newcastle Upon Tyne Hospitals NHS Foundation Trust

\section{Ratna Sohanpal}

Queen Mary University of London

\section{Sarah Saqi-Waseem}

University College London Hospitals NHS Foundation Trust

Moira Kelly

Queen Mary University of London

Hilary Pinnock

The University of Edinburgh

Stephanie Taylor

Queen Mary University of London

\section{Methodology}

Keywords: Depression, Anxiety, Cognitive Behavioural Therapy (CBT), Self-Management, Chronic Obstructive Pulmonary Disease (COPD), Implementation, Intervention development

Posted Date: May 22nd, 2020

DOI: https://doi.org/10.21203/rs.3.rs-29318/v1

License: (c) (1) This work is licensed under a Creative Commons Attribution 4.0 International License.

Read Full License 


\section{Abstract \\ Background}

Healthcare interventions shown to be effective, are commonly then found to be poorly implemented in clinical practice. It is suggested that insufficient attention is given to the implementation process at the developmental phase of intervention. This study outlines a 5 step process for considering both implementation and effectiveness issues from the outset of intervention development. We use the development of a complex intervention Treatment of anxiety and or depression in patients with chronic obstructive disease (TANDEM) to illustrate this process.

\section{Methods}

Intervention development built upon the Medical Research Council framework for developing complex interventions and the Person Based Approach to development of behavioural interventions. Evidence synthesis, stakeholder engagement, qualitative data collection and pre-piloting were all critical steps in intervention development.

\section{Results}

Contact with experts in the field ensured efficiency of design. Qualitative work suggested guiding principles for the intervention such as introducing mood in relation to breathlessness, and providing flexible tailoring to patients' needs, whilst implementation principles focused on training selected respiratory professionals and requiring supervision to ensure standards of care. Subsequent steps of intervention development, pre-piloting and intervention refinement led to an intervention that was deemed acceptable and if successful will be ready for implementation.

\section{Conclusions}

By following the 5-step process recommended, both effectiveness and implementation concerns can be addressed in developing health care interventions, with the likelihood that such interventions will have a more rapid translation in to health care benefits, if shown to be effective.

\section{Contributions To The Literature}

- We describe a five-step process to support intervention developers in considering both effectiveness and implementation strategies throughout the development process.

- A complex psychological intervention for people with COPD (TANDEM) is used as a case study to illustrate the process. 
- The approach builds on and integrates MRC complex intervention framework and the Person Based Approach to intervention development, and includes both health care professional and patient input from the outset to ensure a focus on implementation.

- The study Illustrates how a pre-pilot trial can be used to promote effectiveness and develop implementation strategies prior to formally piloting the trial processes.

\section{Background}

In recent decades the science of developing complex interventions has made significant strides forwards with publication of guidelines and frameworks (1-3). Recognised methods for categorising and describing interventions have become available e.g. TIDieR (4) and WIDER (5) and most recently, for describing intervention development GUIDED (6). These methods are recommended with a view to enabling replication by healthcare services. Despite this there is commonly a gap between an intervention being shown to be clinically and cost effective and its implementation into practice $(7,8)$. This is increasingly leading to multiple research phases where an effectiveness trial is followed by development and evaluation of implementation strategies $(9,10)$.

Improving implementation is important, but this trajectory of stepwise development is costly, inefficient, and may delay attainment of beneficial health outcomes. Recent guidelines therefore recommend consideration of implementation issues at the outset when developing complex interventions (11), but little guidance is given on how to integrate implementation considerations alongside efficacy or effectiveness features which still predominate in intervention development.

The current article uses the development of a complex intervention, TANDEM (Tailored intervention for ANxiety and DEpression Management) for patients with moderate to very severe chronic obstructive pulmonary disease (COPD) and mild to moderate anxiety and/or depression, to illustrate how implementation can be considered alongside initial development of an intervention. We aimed to optimise not only effectiveness but also implementation for the intervention if shown to be effective.

\section{Managing Chronic Obstructive Pulmonary Disease (COPD)}

COPD has a global prevalence of $11.7 \%$ in adults aged over 30 years (12) and is associated with substantial morbidity and mortality (13) with indications that it will be among the top three causes of death by 2030 (14). Progressive reduction in lung function and increased breathlessness affect the physical, social and emotional worlds of patients (15). People with COPD typically have multi-morbidity, including psychological conditions (16-18), such as anxiety and depression, which have a major influence on quality of life $(19,20)$. Anxiety is reported across all ranges of COPD severity, with cited prevalence ranging from 10 to $50 \%(21,22)$. The prevalence of depression (typically around $30 \%$ of all COPD patients $(21,23)$ increases with the severity of COPD $(24)$. Importantly people with COPD and 
anxiety/depression experience more exacerbations, more frequent and longer hospital admissions, and reduced survival $(22,25-28)$.

Pulmonary rehabilitation (PR) is an evidence-based, guideline-recommended intervention for the management of COPD (29), which improves functional capacity, psychological well-being, and quality of life (29-31). However, uptake and reach of PR is less than optimal (32) as practitioners under-refer, and patients fail to attend, or complete, their PR course (33) in part due to their anxiety and/or depression (34). Psychological interventions using a cognitive behavior therapy (CBT) approach either alone, or as a component of PR, have shown promise $(30,35,36)$. However the limited evidence to date, has not considered implementation issues such as reach, or the practicalities of workforce delivery. TANDEM was developed to address this research gap and specifically to design an implementable, cognitive behavioural approach (CBA) intervention, delivered in tandem with $\mathrm{PR}$, aiming to both improve symptoms of anxiety and depression and increase uptake and completion of $P R$, which itself further improves psychological well-being. The protocol for the TANDEM trial is published (37). By CBA we recognize that our intervention does not deliver full CBT but rather draws on the underlying theory to deliver an approach based on CBT. From the outset the process of developing the intervention was understood to be complex, and an open mindset to different guidance and methodologies was applied. This has subsequently been distilled into a five step process which is described below and illustrated in Figure one.

\section{Methods}

Step One - Review of previous evidence and engagement with experts in the area.

Development of TANDEM arose out of a commissioned funding bid for a tailored psychological intervention "combined with physical retraining" for individuals with anxiety and or depression and moderate to severe COPD. Reviews cited within the commissioning brief suggested there was synergy between psychological approaches and physical activity in improving COPD. The study team proposed combining CBA and a supportive self-management intervention with exercise through PR in order to build upon an evidence-based service already embedded within the NHS. It was envisaged from the outset that the intervention would involve both patient-facing components and training of facilitators to deliver the intervention. To ensure we built upon previous evidence we engaged with national investigators who were delivering psychological or self-management interventions with similar aims to TANDEM. A patient and public involvement (PPI) group was set up at this point to provide expertise throughout the whole process of intervention development (38).

Step Two - Identification of theoretical underpinnings and mapping to the intervention

In line with Medical Research Council (MRC) guidance (1) we reviewed the literature to consider whether:

i. CBT was an appropriate underlying theory for the patient-facing element of the intervention and whether any other theoretical approaches should be included 
ii. whether any adult learning theories were appropriate to guide the facilitator training element of the intervention

iii. whether there was an appropriate intervention development approach that should be followed.

In addition, we reviewed the literature on attendance of COPD populations at PR and/or psychosocial interventions to anticipate any issues with implementation and identify theoretical constructs that should be targeted. Theory use was therefore at multiple levels with multiple objectives (e.g. to guide the intervention development process, to inform the intervention, to inform training in delivery of the intervention, to inform implementation). Given this theoretical complexity we aimed to develop a programme theory showing how different approaches could work together and complement each other in the overall TANDEM programme.

Step Three - Qualitative research to understand participant and implementation needs.

Building on the theory and evidence base from steps one and two, understanding 'real life' and implementation challenges to the patients, health care professionals and health systems, was considered essential to inform intervention development. We therefore conducted exploratory qualitative work with both health care professionals and patients with topic guides that addressed three issues:-
i. Difficulties in living with COPD
ii. Opinions on the proposed intervention
iii. Critical elements for successful implementation

We identified respiratory health care professionals (rHCPs) who had an interest in delivery of psychological interventions to patients with COPD through social media and professional networks and invited them to participate in either an individual interview (face to face or telephone) or focus group dependent on participant preference.

We arranged two focus groups for patients and carers. One included COPD patients or carers who had previously experienced CBT (including participants from a specific CBT trial (39); and one with patients and carers attending a Breathe Easy group (a UK support group run by the British Lung Foundation (BLF) who may, or may not, have experienced CBT.

Informed consent to participate and audio-record data was obtained for all participants. Recordings were then transcribed. As the purpose of this qualitative work was to inform intervention development we conducted a rapid thematic analysis informed by a framework approach (40) with the key aim of identifying factors that should be included in the intervention to increase acceptability and ease implementation into routine service if shown to be effective.

Step four - Design of intervention materials 
An intervention working group with responsibility for developing the intervention, was formed including the joint Principal Investigators (PIs), both academic general practitioners (ST, HP), health services researcher (RS), sociologist and qualitative expert (MK), consultant respiratory nurse and cognitive behavioural therapist (KHM), health psychologist (LS), consultant clinical psychologist (SSW) and PPI representative $(\mathrm{CW})$. Formal meetings were convened at three key points.

i. after steps one to three in order to agree guiding principles and basic outline of the intervention and its design

ii. prior to the pre-pilot (step five) to confirm intervention content for both patients and rHCPs

iii. after the pre-pilot (step five) to synthesise qualitative data on acceptability of the intervention, ease of implementation and any refinements required.

In addition, members of the group liaised via e-mail throughout the development process to comment on intervention materials and delivery options. Patient-facing materials were also discussed with PPI colleagues during this development phase to enable on-going patient and carer input. Finally, a reflective intervention development log was kept to ensure the process, considerations and decisions taken were recorded and transparent.

Step Five - Real world pre-pilot phase

Given the complexity and multi-level action of the TANDEM intervention it was felt that even after individual materials had been developed and refined the intervention needed to be delivered as a whole, to understand how elements 'hung' together. This was seen as part of the design phase of the intervention, and an important step prior to testing any research elements (such as randomisation, or outcome data collection) that would occur in a pilot trial (41). We have labeled this step in intervention development as the real world pre-pilot phase. In TANDEM we identified two key questions which needed to be answered before more formal piloting:-

i. Do participants who receive the intervention when delivered by someone already skilled in CBT find it acceptable (i.e. is the potential TANDEM patient intervention appropriate and acceptable) and receive the intervention as intended?

ii. Do participants who receive the intervention when delivered by a novice in a cognitive behavioural approach (CBA) but trained as part of the TANDEM programme find the intervention acceptable and receive it as intended (i.e. is the proposed TANDEM facilitator training sufficient for delivery of the TANDEM patient intervention and consequently implementable)?

A purposive sample of three rHCPs was recruited and trained to deliver the TANDEM intervention, these included one rHCP trained to CBT diploma level, one trained to basic CBT level (i.e. following a three day training external to TANDEM) and one rHCP who had not previously received any CBT training. Once trained these individuals were referred to as "TANDEM facilitators". 
Following methodology used by Heslop-Marshall (42), training for the TANDEM facilitators participating in the real world pre-pilot was conducted over three days, the first two concurrent and the third approximately 6 weeks later to enable practice of skills. To reflect the group nature of future training, clinical members of the research team also joined the three days training as participant observers. Training was delivered by a CBT qualified consultant respiratory nurse (KHM) a health psychologist (LS) and a consultant clinical psychologist (SSW). At the end of each training day all participants were requested to provide verbal feedback on the content and process of training.

Patients with moderate to severe COPD were eligible for participation in the real world pre-pilot phase. Patients received the full 6-8 TANDEM sessions and were invited to interview post-intervention. With participant consent all intervention sessions were audio-recorded. Following delivery of the patient intervention all three of the rHCP TANDEM facilitators were invited to interview. Semi structured interviews were conducted and covered issues such as acceptability and benefit of training, any omissions or improvements, feasibility of delivering the intervention to patients and usefulness of intervention materials.

\section{Results}

\section{Step One - Review of the literature}

Dr Karen Heslop Marshall, a clinical academic agreed to be part of the research collaboration, allowing use of her CBT based Lung Manual (42), which had been evaluated in a successful randomized trial (39), as a basis for the development of the TANDEM intervention. We also recognised the importance of providing high quality, standardised information and education. To enable this, whilst allowing for the majority of our development time to focus on the CBA elements, Professor Sally Singh who had developed a self-management programme the SPACE manual for COPD (43) was invited to join the research collaboration. We therefore used SPACE materials as well as information leaflets from the British Lung Foundation to meet educational and self-management needs.

\section{Step Two - Identification of Theoretical Underpinnings and Mapping to the intervention}

Both The Lung Manual and the SPACE manual are evidence-based and draw on theory. The Lung Manual applied Beck's theory of CBT (44) for managing anxiety and breathlessness in COPD whilst the SPACE manual applied a self-management approach based on Bandura's social learning/cognitive theory (45, 46). Review of other theoretical constructs predictive of outcomes in COPD suggested that Leventhal's Self-Regulation theory (47) was also relevant. Figure two shows the logic model for TANDEM. The basic premise is that how an individual thinks about their COPD (cognitions - including illness and treatment beliefs) influences how they behave (including self-management actions taken) and how they feel (both physical symptoms and emotions). These factors have interactional effects such that depression and /or anxiety can be both reduced or exacerbated depending on the individual's cognitions and behaviours. 
Consequently, by targeting change at a cognitive, behavioural or symptom level this will influence emotional outcomes.

Consultation with Education for Health, a health education charity, and a review of the literature suggested that the pedagogical theory that would be relevant for the training of facilitators was the VARK (Visual, Auditory, Read, Kinesthetic (i.e. experience or practice, simulated or real)) model of learning (48). This ensured that the training would suit individuals with different learning styles.

For intervention development the person-based approach (49) with its focus on using qualitative work to inform guiding principles was considered particularly relevant and therefore guided the intervention development process.

\section{Step Three - Qualitative findings, themes and development of guiding principles}

One focus group comprising six rHCPs (one respiratory consultant, one occupational therapist, three physiotherapists and one exercise practitioner) and seven individual interviews (four psychologists, two physiotherapists and one general practitioner) were conducted. All had experience in working with patients with COPD, either in the community or secondary care. Roles varied, including some with management responsibilities who were able to discuss implementation.

One focus group was held with patients of whom four had COPD, two had other respiratory conditions and two were carers. Very tight timelines precluded formal analysis of transcripts from the patient focus group data, so limiting data to quotes selected from the audio recording. Major local governance delays prevented conduct of the second focus group with patients and their carers who had experience of CBT in time to inform intervention development.

Both patients and professionals presented an overall positive attitude to the idea of the TANDEM intervention:-

And I do think in the long run something like this could be more cost effective and stuff, things like that...I think it would be really useful (rHCP FG002 Occupational therapist)

Themes were developed which related to i) life with COPD, ii) intervention considerations and iii) issues for implementation (see supplementary article 1 for qualitative data summary).

\section{Life with COPD}

All participants including patients and rHCPs recognised depression and anxiety as common in COPD although patients did not always use this terminology. Other issues such as frustration and embarrassment, along with role adjustment and loss, including of social contacts, were common and seen as contributors to mood problems.

"The approach is good...because of all the other thing happening in people's lives which can stop them attending PR and make them anxious and depressed." (focus group patient) 
rHCPs emphasised the challenge of breathlessness to patients with COPD and suggested that discussion and teaching of breathing control early in the intervention is a helpful way to raise issues around mood as well as providing practical help which may increase engagement. There was also recognition that this group may be quite socially isolated and health literacy may vary so the intervention must be accessible to all.

Implementation considerations

The majority of issues for implementation related to the workforce and who would realistically be able to deliver a CBA service. Both rHCPs and psychologists recommended rHCPs to be best placed, however all recognised that there would need to be some selection process and supervision. One clinician was concerned about the cost of the intervention, although others considered that in the long run CBA could be cost-effective.

These themes were subsequently interpreted to provide guiding principles as recommended by the patient based approach to intervention development (49). Table 1 shows these principles and example data extracts.

\section{Step Four: Design And Refinement Of Intervention}

Having agreed the guiding principles for the intervention the intervention working group met to discuss the design of the TANDEM intervention. It was understood that the intervention would be working at two levels i) patient-facing (i.e. CBA delivery) and ii) facilitator-facing (i.e. training programme).

For the patient-facing CBA a range of materials were designed that could

be provided as part of the intervention. These were developed for TANDEM but where possible drew on or used the SPACE manual (43)

handouts. LS developed all materials with iterative refinement from the working group and PPI consultation. Each patient was given a TANDEM folder, in which they could store handouts relevant to them so that individuals had a tailored version of TANDEM materials whilst maintaining consistency in the content provided. In total 6-8 face to face sessions were designed, covering nine topics; those delivered were dependent on individual problems and complexity. Table two provides an overview of TANDEM topics

One topic (dealing with 'other problems') was specifically added as a strategy for keeping the focus of initial sessions on COPD whilst having space later to address issues the person may have outside COPD, for example debt, substance abuse etc. This topic looked at how the CBA, learnt in the context of COPD, could be generalised to different problems, with sign-posting to additional sources of help. At the final 
session discussion was around pulmonary rehabilitation. If there was to be a delay in individuals commencing PR then the facilitator arranged up to nine weekly telephone calls.

The three day facilitator training was provided with a supporting manual which covered the skills needed to deliver TANDEM.

Table 3 details the content of the programme. There was a high level of practical and experiential learning in the group and supportive links within the group were encouraged. Throughout delivery of the CBA intervention facilitators received telephone supervision, one-to-one with senior cognitive behavioural therapists at approximately fortnightly intervals. This on-going supervision was considered to be an integral part of the intervention.

\section{Phase Five - Real World Pre-pilot Study}

All three TANDEM facilitators completed the three training days and two went on to deliver the intervention to three patients (one delivered it to two patients and one to a single patient). The third facilitator (respiratory practice nurse without prior CBT training) did not manage to see any patients due to delays because of research governance and consequent change in work commitments.

\section{TANDEM Facilitators}

After the initial training session, facilitators suggested some changes, specifically an overview of the intervention at the beginning of the training in order to orientate individuals. All the facilitators felt that a role-play activity with a simulated patient (actor), which was conducted as part of the original group training on Day one, was too threatening and at too early a stage of skill development. Instead they requested more demonstrations and more practice with developing formulation.

Interviews at the end of delivering TANDEM to patients, revealed that both facilitators felt the intervention had been well received by participants and feasible to implement, although one had to deliver it over a longer period than scheduled due to patient illness.

Yeah, I mean the two patients who I had were very, very enthusiastic about all elements of the intervention. (PPHCP01)

Generally the facilitators appeared able to follow the manual and found it a helpful guide, but there was questioning of whether someone without previous CBA training would be able to manage:

'I mean section nine, it's got identifying maintenance factors, and it talks about safety behaviours, avoidance and escape, catastrophic interpretation, scanning or hypervigilance, self-fulfilling prophecies, fear of fear, reductions, affectionism, short term rewards. If you're trying to talk to a patient and remember what it says in the manual you might get yourself a little bit flustered.' (PPHCP02) 
One facilitator recommended presenting basic intervention techniques as a toolbox and also the provision of a crib sheet for easy prompting within sessions.

'I feel that people who come away from the training need to have something like a virtual toolbox of techniques that they can refer to ...they expected quite a lot of you... I made myself a crib sheet type of thing' (PPHCP01)

One element that was not adhered to as planned was supervision with a senior psychologist, as the facilitators relied on supervision by an experienced member of their team who was already known to them and who was also part of the study team (KHM). However both facilitators reported this supervision was useful.

\section{Patient perspective}

Patients who had received the TANDEM intervention reported it to be acceptable and beneficial, observing that the facilitators had very good interpersonal skills. There were no substantive suggestions for improvement.

'And then $x$ and I just seemed to get on very well, he's a likeable chap, very laid back. And so it went from there. And then we started doing the things that you asked in TANDEM. Planning... They're just small things, but marvelous' (PPP01, male participant)

\section{Refinements To Tandem After The Pre-pilot Refinements to the TANDEM intervention}

Changes and additions were primarily made to the TANDEM facilitator training, as there were few recommendations for changes to patient materials. These are detailed in Table 3 with additions highlighted in bold. All suggestions were followed: e.g. providing a greater overview of TANDEM at the beginning of session one, keeping to a core set of CBA techniques and outlining a "toolbox" of techniques which could be used. We made video recordings to demonstrate therapeutic skills and CBA techniques. These were made available online, with a facilitator chat facility for ongoing support.

The use of a simulated patient was omitted from the first two days of training and replaced by partnered role-play. The simulated patient role-play was, however, added to the end of day three. Each TANDEM facilitator was individually video-recorded conducting a cognitive behavioural assessment and feed back with the actor. Each video was subsequently assessed by LS and an independent assessor to ensure a minimum standard of competency (see study protocol(37)) was acquired. To enhance learning, and boost confidence, facilitators received one-to-one feedback on their video. A training session on the importance of supervision was added with reflection that supervision is a standard part of psychological training and practice (in contrast to more managerial supervision with which HCPs may be more familiar). 


\section{Refinements to improve implementation}

To improve delivery of the intervention within the trial and future implementation within routine healthcare contexts, five features were added:

i. Facilitators were provided with crib cards for use as prompts within sessions.

ii. An optional session was added for use when a break in sessions had become necessary (e.g. due to patient illness). The aim of this additional session was to refresh topics that had been covered before the break and re-establish current priorities.

iii. Some flexibility in the order of delivery of sessions was allowed reflecting the reality that some patients commenced PR before the end of the TANDEM sessions. It was stipulated that Topics 1-5(or 6) must have been conducted but that if necessary the final topic on expectations of PR could be brought forward as there was no sense in delivering this once PR had started.

iv. A structure for screening potential facilitators including a formal application with a curriculum vitae and telephone interview with one of the principal investigators was developed. The aim was to ensure only fully committed individuals who could meet the study-specific requirements (e.g. flexibility to travel, willingness to complete research modules and good clinical practice training) received training.

v. A booster training session was designed to be delivered to facilitators if there were delays of 3 months or more between initial training and delivery of TANDEM.

For a description of the intervention following TIDieR guidance please see additional file 1.

\section{Discussion}

This paper sets out a five step process for developing the TANDEM intervention that considers both intervention and implementation strategies from the outset to maximise the potential for implementation (if successful) and reduce the time period for translation in to practice. In particular, consideration was given to the workforce that would deliver the intervention and their training and support needs, as well as designing an intervention that could fit structurally into routine clinical care.

Methodologically, we propose use of a novel 'real world pre-pilot' phase within the process of intervention development. This is particularly applicable for complex multi-level or group interventions where the recommended 'think aloud' phase (49) of development may be impractical. The benefits of a pre-pilot phase are to test not only individual elements of an intervention but also how multiple elements of an intervention 'hang together'. This contrasts with traditional feasibility pilot trials, which typically come after intervention development and also test elements of the research process.

Whilst we present a stepped process in reality there was flow between different steps as intervention development is commonly iterative (2). For example, some ideas about the intervention had been considered before the guiding principles were articulated in step three. This was inevitable as our initial 
design was reactive to a funding call (with the additional benefit of enabling peer review comment on our ideas). Importantly, however, at step three we were open to any of our original ideas being challenged and the intervention changed. The intervention working group comprised individuals with diverse backgrounds and were encouraged to reflect and discuss critically.

A challenge we recognised from the outset was that for interventions to be implemented within routine health care services they must be as cost effective as possible. One implication of this is that each patient should receive the optimal intervention for them; one size fits all interventions are likely to be cost inefficient either by under or over treating some individuals. Tailoring has been recommended as a way to address this (7), though it is a significant challenge to design an intervention that is both responsive to each individual's needs yet standardised enough to allow for robust evaluation and assessment of fidelity when implemented. TANDEM attempted to balance standardisation with flexibility by using a modular/topic-based approach where topics are standardised, but which topics are addressed and in what order is dependent on the individual or the circumstances (such as timing of the PR course). The success of tailoring in this way is as yet unknown but will be examined as part of the process evaluation of the trial.

A further challenge was that some aspects of implementation were more difficult because of delivery within the context of a research trial. In our pre-pilot we experienced two illustrations of this. The first was that obtaining local research governance approval caused a delay of 6 months between training TANDEM facilitators and being allowed to deliver the intervention. This was detrimental as it risked loss of confidence amongst the facilitators, skill drift and knowledge decrease during the gap between training and implementation. We would urge research governance frameworks to consider the impact of their processes in the context of intervention development and implementation research. Within a clinical service this might be less likely to occur, but clinical practice has its own challenges such as staff turnover, funding pressures, changes in management etc. The implementation strategies we provided, such as booster training and online support resources to overcome study specific issues, could equally apply in the clinical setting and will be explored within our trial process evaluation.

The second was that our TANDEM facilitators could not be embedded within the participants' clinical team (to prevent 'contamination' of controls (37)) with the results that they would not have support, both practical and emotional, from clinical colleagues. The research team attempted to overcome this by providing facilitators with a clinical support network with the chief investigators ST, HP (both general practitioners) and the consultant respiratory nurse (KHM) was available to answer any concerns. This was in addition to the clinical supervision provided for ensuring therapeutic competence and access to a chat facility with other facilitators on the skills website.

\section{Strengths and Limitations}

A strength of TANDEM is in its multidisciplinary team approach to intervention development. We specifically built upon previous work by collaborating with national experts enhancing the expertise 
available to TANDEM and reducing potential duplication. Surprisingly this has not been explicitly recommended in recent guidance (11) but in our view is an important step in ensuring the most efficient use of limited resources.

A further strength is the systematic and transparent approach to intervention development that we have outlined.

In step three where we conducted qualitative work to highlight important issues for the intervention and its implementation, only a limited number of health care professionals and patients could participate. It may be that important voices such as that of practice nurses, health service managers or commissioners, who could have provided different perspectives for implementation, were not heard. This step, however was scheduled within a relatively rapid time-frame to be useful to inform intervention development, and therefore a more pragmatic approach was needed than would be used for a formal qualitative study.

In the pre-pilot phase only three facilitators and three participants experienced the intervention. Greater numbers would have provided greater feedback however again this was not possible due to resource limitations and logistical requirements of the time-scale required for intervention development.

Resonance of TANDEM with Implementation Research

Although we aimed to address integration of implementation issues throughout our intervention development process we did not explicitly do this in our research design. Methodological advances in implementation research have made recommendations for trial designs known as hybrid designs whereby effectiveness and implementation potential of strategies are investigated concurrently (50). Recently there have also been calls for this within behavioural science (51). Three levels of hybrid design are proposed which vary in the relative balance between the focus on effectiveness versus implementation (50). These designs require a set of assumptions to be met around features of the intervention, such as the level of face validity, strength of the existing evidence base, risks associated with the intervention and implementation momentum. TANDEM meets these assumptions and hence can be considered as similar to a hybrid design:

i. It has high face validity given CBT and pulmonary rehabilitation are well recognised and guidelinerecommended interventions.

ii. PR has an established evidence base for COPD (29-31) and CBT is showing promise for COPD patients(35, 39).

iii. Few risks have been described in evaluations of CBT or PR and there is little reason to expect increased risk through the integration of these approaches such as proposed in TANDEM;

iv. Importantly there is a current implementation momentum within the clinical system towards both implementation of PR (52), and treatment of psychological issues within chronic illness, in the UK (53). Furthermore recent policy has advocated the expansion of current primary care mental health 
services (Integrated Access to Psychological Therapies (IAPT)) to work with patients with COPD (54).

Future studies developing interventions for implementation may wish explicitly to consider a greater diversity of research design, including hybrid designs to see if this can facilitate implementation.

\section{Conclusions}

We recommend that intervention developers play greater consideration to implementation issues both at the early stages and throughout the intervention development phase. We outline five steps focused around understanding and working with prior knowledge, using theory, exploring the needs of the target groups, developing prototypes and testing within a real world pre-pilot, all of which can consider implementation issues as needs as well as that of intervention content. By conducting this work within a framework of critical reflectivity we suggest more successful and implementable interventions are likely to be developed.

\section{Abbreviations}

\section{BLF}

British Lung Foundation

CBA

Cognitive Behavioural Approach

CBT

Cognitive Behaviour Therapy

COPD

Chronic Obstructive Pulmonary Disease

GUIDED

GUIDance for the rEporting of intervention Development

IAPT

Integrated Access to Psychological Therapies

MRC

Medical Research Council

PPI

Patient and Public Involvement

PR

Pulmonary Rehabilitation

rHCP

respiratory Health Care Professional

TANDEM

Tailored intervention for ANxiety and DEpression Management

TIDieR 
Template for Intervention Description and Replication

VARK

Visual Auditory Reading Kinesthetic

WHO

World Health organization

WIDER

Workgroup for Intervention Development and Evaluation Research

\section{Declarations}

Ethics approval and consent to participate

The study (pilot and main trial) was approved by the London-Queen Square Research Ethics Committee, reference 17/LO/0095. Informed consent was obtained from all study participants.

Consent for publication

Consent for publication was received from all participants before their commencement in the study.

Availability of data and materials

Data generated and analysed during this study is included in this published article. Study manuals and training materials are copyrighted but will be available upon reasonable request to the authors.

Competing interests

ST is the chief investigator or co-investigator on multiple previous and current research grants from the UK National Institute for Health Research and CRC UK.

KM received funding from NIHR for a PhD study on CBT in COPD, received honoraria from pharmaceutical companies for presenting on CBT in COPD, I have received travel expenses for speaking at conferences and professional organisations on CBT and I am a director of Pivotal Health Company which provides CBT training for healthcare professionals in the physical health setting.

Funding

This report is independent research supported by the National Institute for Health Research Health Technology Assessment programme (project number 13/146/02).

ST is supported by the National Institute for Health Research ARC North Thames. The views expressed in this publication are those of the authors and not necessarily those of the National Institute for Health Research, the NHS or the Department of Health and Social Care.

Authors' contributions 
ST and HP conceived the study. LS, KHM, SSW were responsible for the primary development of the intervention. All authors participated in the intervention development steering group. RS contributed to the collection of qualitative data and MK advised on analysis of qualitative data. All authors made substantial contributions to the design of the study. All authors have helped to draft this manuscript and/or revised it and all have read and approved the final manuscript.

Acknowledgements

The authors are grateful for the support of Mr. Chris Warburton and all our other Public Involvement advisors; and our Trial Steering Committee and Data Monitoring and Ethics Committee members.

\section{References}

1. Craig P, Macintyre S, et al. Developing and evaluating complex interventions: the new Medical Research Council guidance. BMJ. 2008;337.

2. O'Cathain A, Croot L, Sworn K, Duncan E, Rousseau N, Turner K, et al. Taxonomy of approaches to developing interventions to improve health: a systematic methods overview. Pilot Feasibility Studies. 2019;5:41.

3. Michie S, West R. The behaviour change wheel: a new method for characterising and designing behaviour change interventions. Implement Sci 2011;23(6).

4. Hoffmann T, Boutron I, et al. Better reporting of interventions: template for intervention description and replication (TIDieR) checklist and guide. BMJ. 2014;348.

5. Albrecht L, Arseneau D, Scott S. Development of a checklist to assess the quality of reporting of knowledge translation interventions using the Workgroup for Intervention Development and Evaluation Research (WIDER) recommendations. Implement Sci. 2013;8.

6. Duncan E, Rousseau N, Croot L, Sworn K, Turner KM, Yardley L, Hoddinott P. Guidance for reporting intervention development studies in health research (GUIDED): an evidence-based consensus study. BMJ open. 2020. Epub Apr 8;10(4):e033516.

7. Taylor S, Epiphaniou E, Pearce G, Parke H, Schwappach A, Purushotham N, Jacob S, Griffiths CJ, Greenhalgh T. \& Sheikh A. A rapid synthesis of the evidence on interventions supporting selfmanagement for people with long-term conditions (PRISMS Practical systematic Review of SelfManagement Support for long-term conditions). Health Services Delivery Research. 2014;2:53.

8. Pinnock $H$, Parke $H$, Panagioti $M$, et al. Systematic meta-review of supported self-management for asthma: a healthcare perspective. BMC Med. 2017;15(64).

9. Pinnock H, Sheikh A. Standards for reporting implementation studies (StaRI): enhancing reporting to improve care. NPJ primary care respiratory medicine. 2017 Jun 26;27(1):42.

10. Peters $\mathrm{D}$, Alonge $\mathrm{O}$, Agyepong I, Tran N. Implementation research: what it is and how to do it. BMJ. 2013;347:6753. 
11. O'Cathain A, Croot L, Sworn K, Duncan E, Rousseau N, Turner K, et al. Guidance on how to develop complex interventions to improve health and healthcare 2019 BMJ Open. 9(18).

12. Adeloye D, Lee C, Basquill C, Papana A, Theodoratou E, Nair H, Gasevic D, Sridhar D, Campbell H, et al. Global and regional estimates of COPD prevalence: systematic review and meta-analysis. J Glob Health. 2015;5(2).

13. Soriano J, Abate KH, Abera SF, Agrawal A, Ahmed MB, Aichour AN, et al. Global, regional, and national deaths, prevalence, disability-adjusted life years, and years lived with disability for chronic obstructive pulmonary disease and asthma, 1990-2015: a systematic analysis for the Global Burden of Disease Study 2015. Lancet Respir Med. 2017;5(9):691-706.

14. WHO. Chronic obstructive pulmonary disease Burden of Disease: 2019.

15. Tsiligianni I, Kocks J, Tzanakis N, Siafakas N, van der Molen T. Factors that influence diseasespecific quality of life or health status in patients with COPD: a review and meta-analysis of Pearson correlations. Primary care respiratory journal. 2011;Sep;20(3):257-68.

16. Department of Health. An outcomes strategy for COPD and asthma: NHS Companion Document. In: Department of Health 2012 Accessed 8 Oct 2019. Available from: https://http://www.gov.uk/government/publications/an-outcomes.

17. Wong CJ, Marciniuk DD, Rennie D. Fatigue in patients with COPD participating in a pulmonary rehabilitation program. Int J Chron Obstruct Pulmon Dis. 2010;5:319-26.

18. Cassidy S, Gardani M, Kirkwood K. Attendance at pulmonary rehabilitation classes: an exploration of demographic, physiological and psychological factors that predict completion of treatment. Chron Respir Dis. 2014;11(2):95-102.

19. de Voogd JN, Postema K, van Sonderen E, Ranchor AV, Coyne JC, Sanderman R. More evidence that depressive symptoms predict mortality in COPD patients: is Type $\mathrm{D}$ personality an alternative explanation? Ann Behav Med. 2009;38(2).

20. Papaioannou Al, Tsikrika S, Karakontaki F, Kastanakis E, Banya W, Haniotou A, Papiris S, Loukides S, Polychronopoulos $\mathrm{V}$, et al. The impact of depressive symptoms on recovery and outcome of hospitalised COPD exacerbations. Eur Respir J. 2013;41(4):815-23.

21. Baxter N, Holzhauer-Barrie J, Robinson S, Stone P, Quint J, Roberts CM. Planning for every breath. National Chronic Obstructive Pulmonary Disease (COPD) Audit Programme: Primary care audit (Wales) 2015-17. National Report. 2017.

22. Yohannes AM, Alexopoulos GS. Depression and anxiety in patients with COPD. European respiratory review; 2014 Sep;23(133):345-9.

23. Bock K, Hilberg O, Løkke A. Screening tools for evaluation of depression in Chronic Obstructive Pulmonary Disease (COPD). A systematic review. Eur Clin Respir J. 2017;4(1).

24. Cafarella PA, Usmani Z, Frith PA. Treatments for anxiety and depression in patients with chronic obstructive pulmonary disease: a literature review. Respirology. 2012;17(4):627-38.

25. Ng TP, Tan WC, Cao Z, Ong KC, Eng P. Depressive symptoms and chronic obstructive pulmonary disease: effect on mortality, hospital readmission, symptom burden, functional status, and quality of 
life. Arch Intern Med. 2007;167(1):60-7.

26. Eisner MD, Yelin EH, Katz PP, Sanchez G, Iribarren C, Omachi TA. Influence of anxiety on health outcomes in COPD. Thorax. 2010;65(3):229-34.

27. Laurin C, Bacon SL, Lavoie KL. Impact of anxiety and depression on chronic obstructive pulmonary disease exacerbation risk. Am J Respir Crit Care Med. 2012;185(9):918-23.

28. Dickens C, Blakemore A, Khara A, McGowan L, Tomenson B, Jackson J, Walker L, Guthrie E. Does depression predict the use of urgent and unscheduled care by people with long term conditions? A systematic review with meta-analysis. J Psychosom Res. 2012;73(5):334-42.

29. McCarthy BCD, Devane D, Murphy K, Murphy E, Lacasse Y. Pulmonary rehabilitation for chronic obstructive pulmonary disease. Cochrane Database of Systematic Reviews. 2015;2:CD003793.

30. Coventry PA, Hind D. Comprehensive pulmonary rehabilitation for anxiety and depression in adults with chronic obstructive pulmonary disease: Systematic review and meta-analysis. J Psychosom Res. 2007 Nov;63(5):551-65.

31. Steiner MC, Roberts CM. Pulmonary rehabilitation: the next steps. Lancet Resp Med. 2016 Mar;4(3):172-3.

32. McNamara RJ, Kearns R, Dennis SM, Gardner MFH, McDonald K, Knowledge J. Skill, and Confidence in People Attending Pulmonary Rehabilitation: A Cross-Sectional Analysis of the Effects and Determinants of Patient Activation. Journal of patient experience. 2019 Jun;6(2):117-25.

33. Steiner M, Lowe D, Searle L, Skipper E, Welham S, Roberts CM. Pulmonary Rehabilitation: time to breathe better. National Chronic Obstructive Pulmonary Disease (COPD) Audit Programme: resources and organisation of pulmonary rehabilitation services in England and Wales 2015.

34. Sohanpal R, Steed L, Mars T, Taylor SJ. Understanding patient participation behaviour in studies of COPD support programmes such as pulmonary rehabilitation and self-management: a qualitative synthesis with application of theory. NPJ primary care respiratory medicine. 2015 Sep 17;25:15054.

35. Pollok J, Esterman AJ, Carson-Chahhoud KV. Psychological therapies for the treatment of depression in chronic obstructive pulmonary disease.. Cochrane Database of Systematic Reviews. 2019 (3):CD012347.

36. Smith S, Ketcheson L, Larson JL. A review of the effectiveness of psychological interventions used for anxiety and depression in chronic obstructive pulmonary disease. BMJ Open Respir Res. 2014;1(1):e000042.

37. Sohanpal R, Pinnock H, Steed L, Heslop Marshall K, Chan C, Kelly M, et al. Tailored, psychological intervention for anxiety or depression in people with chronic obstructive pulmonary disease (COPD), TANDEM (Tailored intervention for ANxiety and DEpression Management in COPD): protocol for a randomised controlled trial. Trials. 2020 Jan 6;21(1):18.

38. Jackson T. Pinnock H, Worth A. Patient and Public involvement in research: from tokenistic box ticking to valued team members. BMC Medicine. 2020;18.

39. Heslop-Marshall K, Carrick-Sen D, Newton J, Echevarria C, Stenton C, Jambon M, Gray J, Pearce K, Burns G, et al. Randomised controlled trial of cognitive behavioural therapy in COPD. ERJ Open Res. 
2018;4(4):00094-2018.

40. Gale NK, Heath G, Cameron E, et al. Using the framework method for the analysis of qualitative data in multi-disciplinary health research. BMC Med Res Methodol. 2013;13:117.

41. Eldridge SM, Campbell MJ, Thabane L, Hopewell S, Coleman CL, et al. Defining Feasibility and Pilot Studies in Preparation for Randomised Controlled Trials: Development of a Conceptual Framework. PLoS ONE. 2016;11(3):e0150205.

42. Heslop K, Baker C, Burns G, Carrick-Sen D, De Soyza A. Effectiveness of cognitive behavioural therapy (CBT) interventions for anxiety in patients with chronic obstructive pulmonary disease (COPD) undertaken by respiratory nurses: the COPD CBT CARE study. BMC pulmonary medicine. 2013;13.

43. Apps LD, Harrison SL, Sewell L, Williams JE, Young HM, et al. The development and pilot testing of the Self-management Programme of Activity, Coping and Education for Chronic Obstructive Pulmonary Disease (SPACE for COPD). International Journal of COPD. 2013;8:317-27.

44. Beck AT. The diagnosis and management of depression. Philadelphia: University of Pennsylvania Press; 1967.

45. Bandura A. Social Learning Theory. Englewood Cliffs: Prentice Hall; 1977.

46. Bandura A. Self-efficacy: the exercise of control. New York:NY: Freeman; 1997.

47. Leventhal H. Representations, procedures and affect in illness self regulation. In: Baum ART, Singer J, editors. Handbook of Health Psychology. New York: Erlbaum; 1997.

48. Fleming N. Helping Students Understand How They Learn. The Teaching Professor. 1992;7(4).

49. Yardley L,. Arden-Close E, Muller I. The person-based approach to enhancing the acceptability and feasibility of interventions. Pilot feasibility studies. 2015;1:37.

50. Curran GM, Bauer M, Mittman B, Pyne JM, Stetler C. Effectiveness-implementation hybrid designs: combining elements of clinical effectiveness and implementation research to enhance public health impact. Med Care. 2012;Mar;50(3):217-26.

51. Luszczynska A. It's time for effectiveness- implementation hybrid research on behaviour change. Health Psychology Review. 2020. DOl:10.1080/17437199.2019.1707105.

52. RCP. National Asthma and COPD Audit Programme (NACAP). pulmonary rehabilitation workstream 2018 [cited 2020 8th May]. Available from: https://http://www.rcplondon.ac.uk/projects/nationalasthma-and-copd-audit-programme-nacap-pulmonary-rehabilitation-workstream.

53. Department of Health. The NHS long term plan 2019. Available from: https://http://www.longtermplan.nhs.uk.

54. National Collaborating Centre for Mental Health. The Improving Access to Psychological Therapies (IAPT) Pathway for People with Long-term Physical Health Conditions and Medically Unexplained Symptoms. Full implementation guidance. London: National Collaborating Centre for Mental Health; 2018.

\section{Tables}


Table 1

Guiding Principles for TANDEM

Intervention Guiding Principles Data Extract

Depression and anxiety are key topics but could be introduced via breathlessness
I think they most often talk about symptoms like breathlessness, rather than saying that they're anxious or depressed. (HCP006 Physio) "terminology is important" such as "dealing with" "living with" (patient)

The Intervention should be tailored/flexible to individuals

Sessions could be offered at home, or clinic but there may be limitations to the latter, accessibility is key

Clear expectations and boundaries should be set at the start of the intervention

Implementation Guiding Principles

Delivery by Respiratory

Professionals rather than psychologists is preferable

Some selection and training of facilitators will be needed

Supervision of facilitators delivering the intervention is essential and should be ongoing

The intervention must be deliverable and supported by management

The intervention should be able to account for patient breaks due to illness it's just that patients are all different, and therefore present very differently and the intervention has to be tailored individually to what they're presenting with. (HCP003, psychologist)

So I think having the capacity to start off at home is certainly a good idea. I think just something about accessible locations. (HCP002, Psychologist)

So there needs to be quite clear boundaries about what the intervention offers and doesn't offer.(HCP006, physio)
Illustrating quotes

"it feels important that other members of the healthcare team are being trained up in these approaches. That can only be a good thing.... (HCP003,psychologist)

A lot of people would be attracted to this, but it's not for everyone to deliver.(HCP005, physio)

'What training would this nurse have?' (Patient)

I think that's important.[supervision] (HCP005, physiotherapist)
'There's no point evaluating it if it's not something that's going to be deliverable. (HCP FG001 Doctor) ....that's important, and you have to acknowledge if they're not feeling well, we have to assess it and make sure that they get the right treatment..(HCP005, Physiotherapist)

'Timing is important' (patient) 
Table 2

Summary of TANDEM intervention (patient facing) content

\begin{tabular}{|c|c|c|}
\hline & Topics Covered & Content \\
\hline $\begin{array}{l}\text { Session } \\
1\end{array}$ & $\begin{array}{l}\text { Introduction, } \\
\text { setting } \\
\text { expectations } \\
\text { Topic 1- What is } \\
\text { COPD? } \\
\text { Topic } 2 \text { - Taking } \\
\text { control of COPD } \\
\text { Topic } 3 \text { - The } \\
\text { patient experience } \\
\text { of breathlessness }\end{array}$ & $\begin{array}{l}\text { Eliciting the patients understanding of COPD, identifying and } \\
\text { working with illness and treatment beliefs and acceptance. } \\
\text { Teaching basic breathing control. }\end{array}$ \\
\hline $\begin{array}{l}\text { Session } \\
2\end{array}$ & $\begin{array}{l}\text { Feedback from } \\
\text { home practice } \\
\text { Topic } 4- \\
\text { Introducing mood } \\
\text { and COPD }\end{array}$ & $\begin{array}{l}\text { Conducting a formulation and presentation of a cognitive } \\
\text { behavioural approach }\end{array}$ \\
\hline $\begin{array}{l}\text { Session } \\
3-7\end{array}$ & $\begin{array}{l}\text { Feedback from } \\
\text { home practice } \\
\text { Topic } 5 \text { - } \\
\text { Managing Anxiety } \\
\text { and COPD } \\
\text { Topic } 6 \text { - } \\
\text { Managing } \\
\text { Depression and } \\
\text { COPD } \\
\text { Topic } 7 \text { - Applying } \\
\text { the CBA to other } \\
\text { problems } \\
\text { (optional) }\end{array}$ & $\begin{array}{l}\text { Up to four sessions to conduct cognitive behavioural work on } \\
\text { anxiety and/or depression dependent on individual need. One } \\
\text { further session available to discuss other problems if needed. }\end{array}$ \\
\hline $\begin{array}{l}\text { Session } \\
5-7\end{array}$ & $\begin{array}{l}\text { Feedback from } \\
\text { home practice } \\
\text { Topic } 8 \text { - Living } \\
\text { with COPD day to } \\
\text { day }\end{array}$ & $\begin{array}{l}\text { Self-management approaches to COPD. Learning to problem solve } \\
\text { and set goals. }\end{array}$ \\
\hline $\begin{array}{l}\text { Session } \\
6-8\end{array}$ & $\begin{array}{l}\text { Feedback from } \\
\text { home practice } \\
\text { Topic } 9- \\
\text { Preparing for } \\
\text { Pulmonary } \\
\text { Rehabilitation }\end{array}$ & Expectations of PR, addressing worries and concerns \\
\hline
\end{tabular}


Table 3

Overview of TANDEM facilitator training.

\begin{tabular}{|c|c|c|}
\hline Day One & Day Two & Day Three \\
\hline $\begin{array}{l}\text { - Introductions } \\
\text { - TANDEM overview } \\
\text { - The patients experience of COPD (group } \\
\text { exercise) } \\
\text { - What are Depression and Anxiety? (group } \\
\text { exercise) } \\
\text { - Depression and anxiety in COPD } \\
\text { - Introduction to CBA (group exercise) } \\
\text { demo therapeutic skills (video } \\
\text { - Making an assessment - recognizing } \\
\text { thoughts, feelings behaviours, symptoms } \\
\text { (practical) } \\
\text { - Sharing ideas with patients (practical) } \\
\text { - Feedback on worries and concerns after day } \\
\text { one }\end{array}$ & $\begin{array}{l}\text { - CBA techniques } \\
\text { (practical) } \\
\text { o Psychoeducation } \\
\text { o Breathing control } \\
\text { o Distraction } \\
\text { o Monitoring } \\
\text { o Problem Solving } \\
\text { o Goal setting } \\
\text { o Graded } \\
\text { practice/simple } \\
\text { behavioural experiments } \\
\text { o Challenging Thoughts } \\
\text { (video demonstration) } \\
\text { - Toolbox for Anxiety } \\
\text { - Toolbox for Depression } \\
\text { - Preparation for case } \\
\text { studies }\end{array}$ & $\begin{array}{l}\text { - Case study feedback } \\
\text { - Individual practice with } \\
\text { actor (videoed) } \\
\text { - Delivering TANDEM } \\
\text { session by session } \\
\text { including } \\
\text { o Changing behavior } \\
\text { o Preparing for PR } \\
\text { (using a photobook) } \\
\text { - Importance of } \\
\text { Supervision } \\
\text { - Risk Assessment } \\
\text { - Research } \\
\text { Requirements } \\
\text { Provision of crib cards }\end{array}$ \\
\hline
\end{tabular}

\section{Figures}




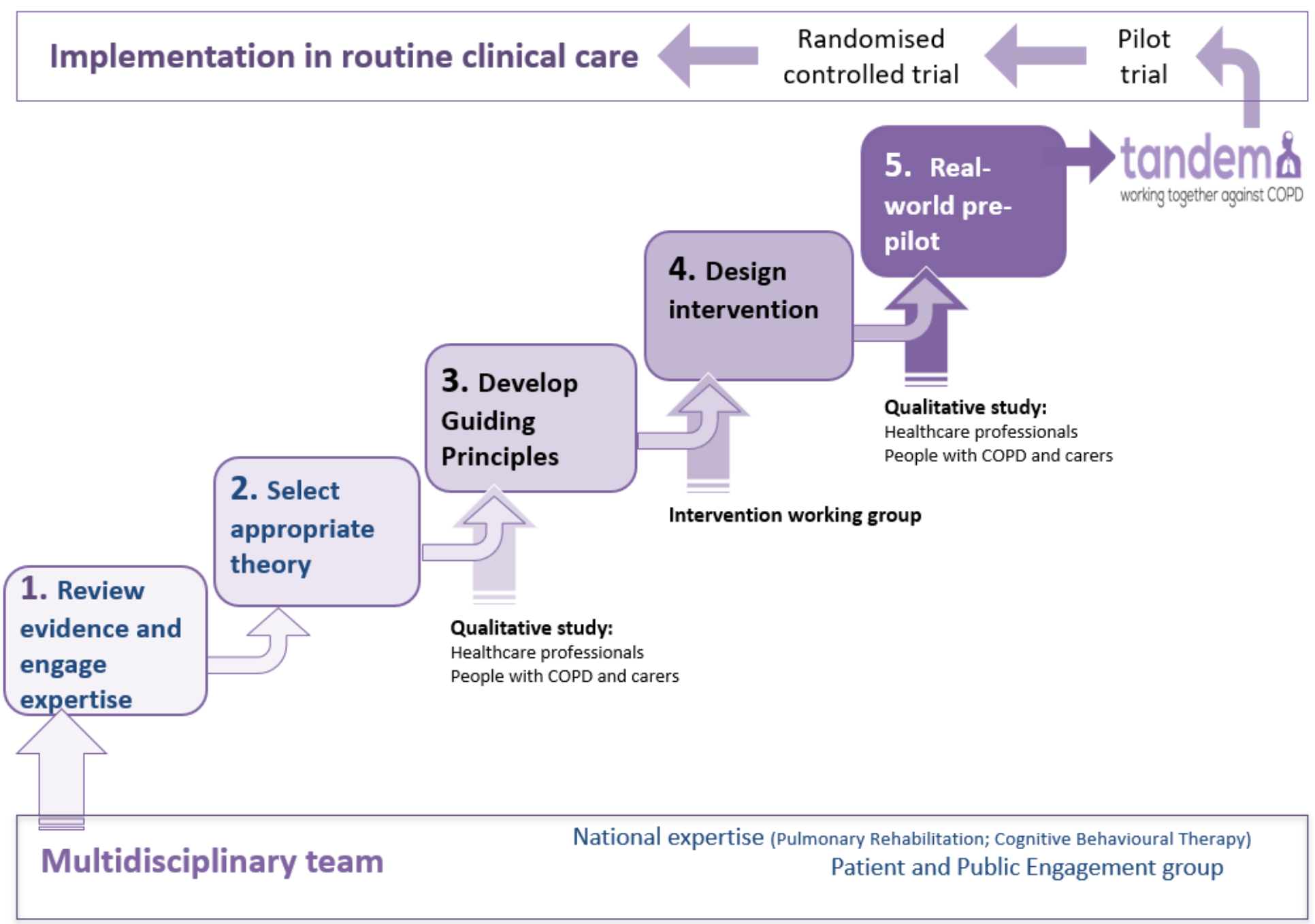

Figure 1

Schema for TANDEM Intervention Development Process 


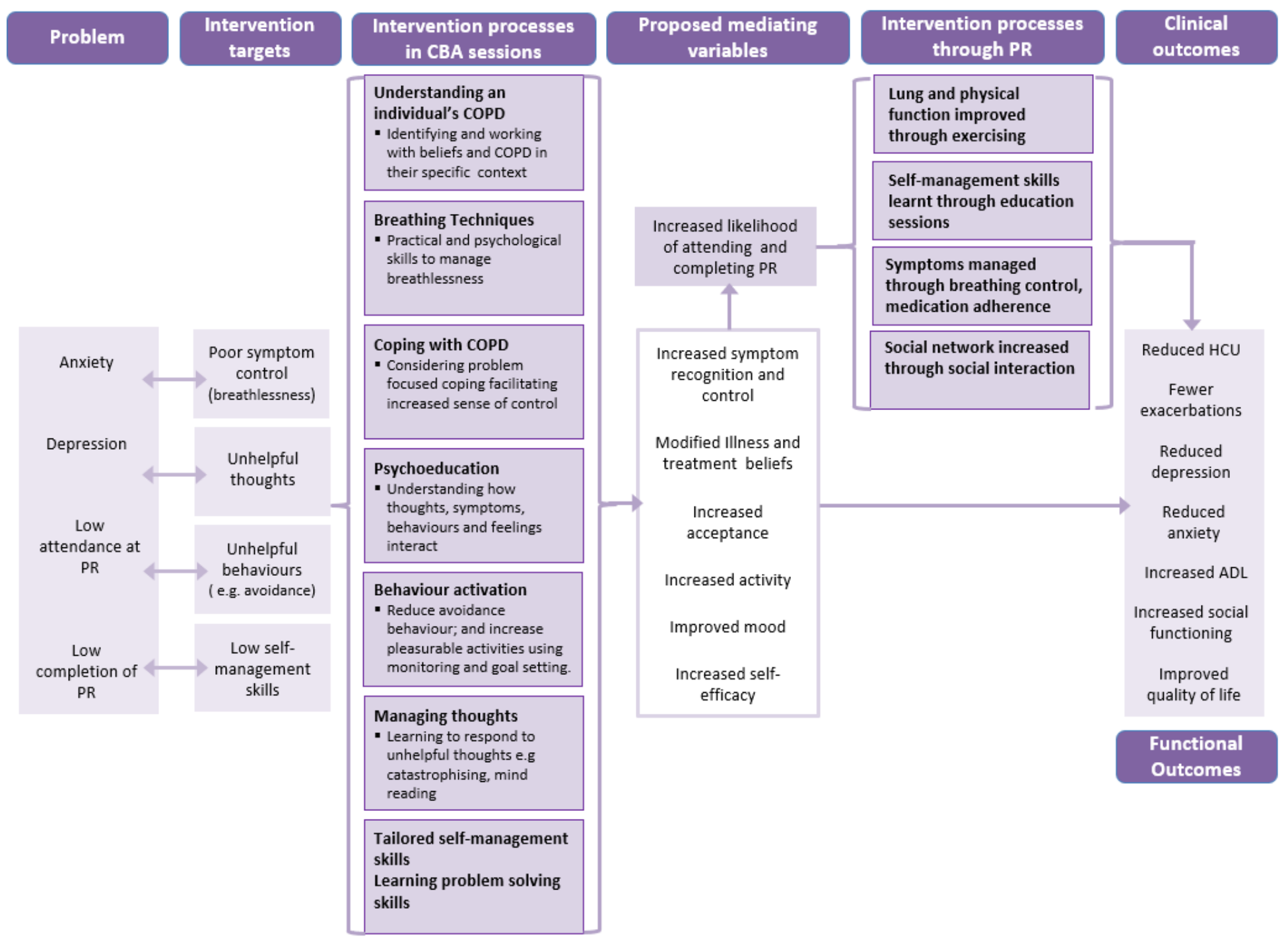

Figure 2

Logic Model for TANDEM Intervention

\section{Supplementary Files}

This is a list of supplementary files associated with this preprint. Click to download.

- STEEDGUIDEDPG1.pdf

- STEEDGuidedpg2.pdf

- STEEDGUIDEDPG1.pdf

- STEEDGuidedpg2.pdf 